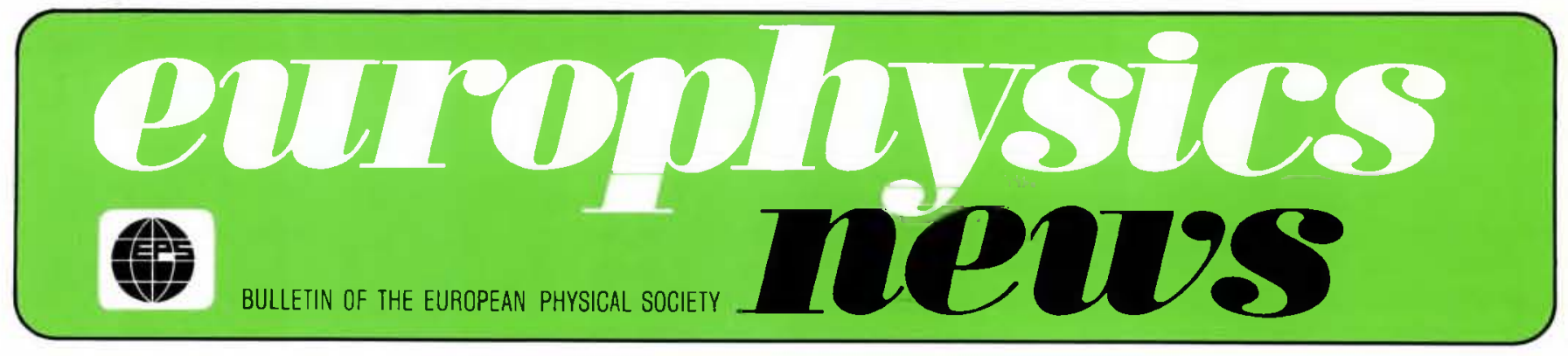

\title{
Klaus von Klitzing
}

\section{NOBEL PRIZE IN PHYSICS}

\section{Yoseph Imry, Tel Aviv}

(School of Physics and Astronomy, Tel Aviv University)

An October 16, 1985, the Swedish Academy of Sciences announced the award of the 1985 Nobel Prize in Physics to Klaus von Klitzing of the Max-Planck Institute in Stuttgart, for the discovery of the Quantized Hall Effect (QHE). The QHE was reported in 1980 in a paper $\left.{ }^{1}\right\}$ by von Klitzing in collaboration with $\mathrm{G}$. Dorda of Siemens and $M$. Pepper of Cambridge. As shown in Fig. 1, the Hall conductance $G_{x y}$ of a $2 \mathrm{D}$ electron gas in an MOS was found, for finite ranges of the gate voltage in which the regular conductance, $G_{x x}$, was severely reduced, to be quantized with plateaux having

$G_{x y}=j h / e^{2} \cong J \cdot(25812.80 \Omega)^{-1}$. $j$ integer

i.e. integer multiples of a basic quantum unit. The initial accuracy of $\cong 10^{-6}$ of this macroscopic quantum effect has meanwhile been surpassed by an order of magnitude and the result was found to be universal - independent of material and sample properties. The plateaux were also observed by Kawaji and Wakabayashi ${ }^{2}$ ).

Besides its sheer beauty, this striking result touches upon several topics of fundamental interest, has important applications to precision measurements and metrology, has motivated and will undoubtedly lead to novel developments. Moreover, it relies on the physics of devices of great industrial interest ${ }^{3}$ ).

Before explaining the effect briefly and reviewing its applications and implications, we remark that this was a truly experimental discovery, found through a measurement of, in principle, a routine character, except, of course, for

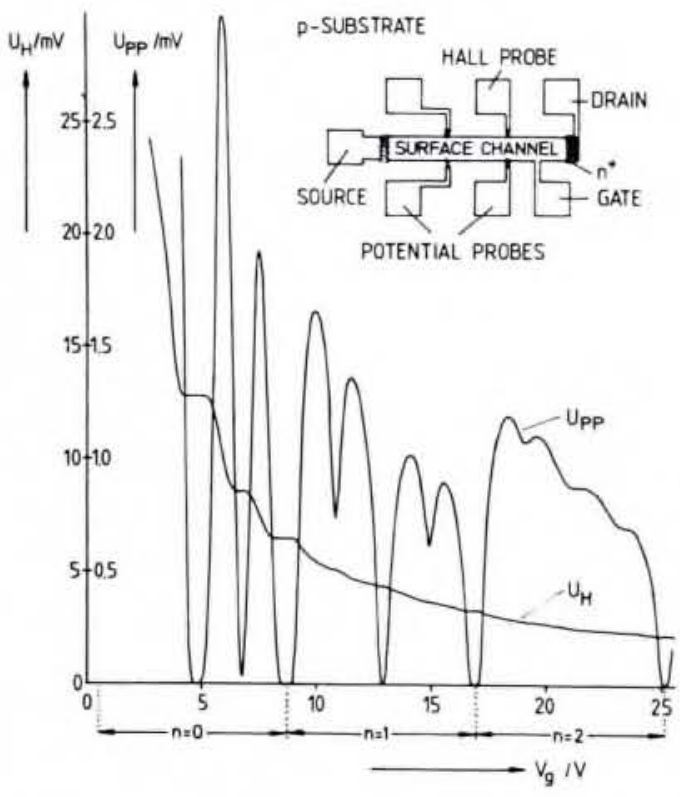

Fig. 1 - The Hall voltage, $U_{H}$ and the voltage parallel to the current $U_{p o}$ for the device shown in the inset as a function of the gate voltage with $T=1.5$ $K, B=18 \mathrm{~T}$. Whenever $U_{p P}$ is strongly depressed $U_{H}$ has a plateau with a quantized value of $G_{x y}$.

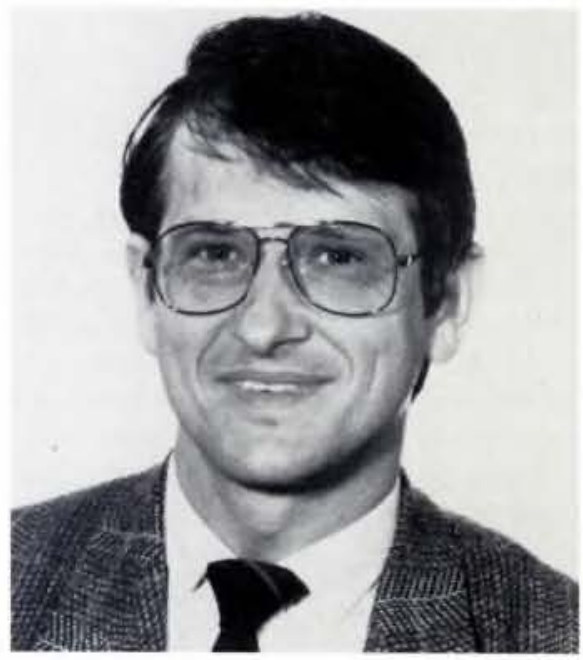

Klaus von Klitzing now aged 42 began his physics studies at the Technical University in Braunschweig and then in 1972 moved to the University of Würzburg gaining his Habilitation in 1978 after spending a period at the University of Oxford. He was able to continue with his research at Würzburg through being awarded a Heisenberg Stipend. Then as guest of the Max-Planck-Institut in Stuttgart he began experiments at the joint MPICNRS high magnetic field laboratory in Grenoble where he made his now celebrated measurements. Von Klitzing is presently full time with the Max-Planck-Institut für Festkörperforschung in Stuttgart having been for a short time Associate Professor at the Technical University in Munich.

\section{Contents}

Klaus von Klitzing, 1985 Nobel Prize Winner

Message from the CMD

Optical Fibre Technology at STL

Cumulative Index, 1981-1985

Physics of the Ocean

Graphite Intercalation

Compounds

New EPS Members

Collaboration with the APS

Europhysics Letters

1
3
4
9
13
16
19
20
20


the high magnetic field $(\sim 15 T)$ and the high quality MOS used. The experiment has not required "big physics" equipment or machinery. Also, while there had been a theoretical calculation yielding approximately such an effect ${ }^{4}$ ), the conditions under which it was really observed were outside the range of validity of that theory. Thus, the QHE discovery (as well as subsequent ones ${ }^{5}$ ), did take theoreticians by surprise!

\section{Explanations}

The initial discovery employed a MOSFET (metal-oxide-semiconductorfield-effect-transistor), where a $2 \mathrm{D}$ electronic layer $\left.{ }^{6}\right)$ is formed at the interface between, e.g. p-type silicon and a $10^{3} \AA$ layer of $\mathrm{SiO}_{2}$, in a strong electric field perpendicular to the interface. The latter is controlled by a gate voltage applied between the $\mathrm{Si}$ and a metallic layer deposited on top of the $\mathrm{SiO}_{2}$. It was first demonstrated in Ref. 7 and is now well established both experimentally and theoretically ${ }^{6}$ ) that owing to the quantization of the electronic levels perpendicular to the interface and the possibility of controlling the electron density by the gate voltage, conditions where all electrons reside in the lowest such level can be achieved. The electronic motion is then predominantly $2 \mathrm{D}$, parallel to the interface. One interesting aspect of an ideal 2D system is that the motion in these parallel dimensions can be fully quantized in a strong magnetic field, $B$, into discrete Landau levels having energies $(n+1 / 2) \hbar \omega_{c}$, with $\hbar \omega_{c}=e B / m^{*} c$, $m^{*}$ being the effective mass of the 2D motion. Other $2 \mathrm{D}$ electron systems are available, notably in quantum wells formed at the interface between two ap propriately grown semiconductors $\left.{ }^{5,6}\right)$. The QHE has by now been observed in several of those as well.

The quantum unit of conductance, $h / e^{2}$, is quite interesting in itself. In classical physics, a natural unit for conductance is provided by the velocity of light in vacuum, $c$, as the impedance of free space is $4 \pi / c \cong 376.7 \Omega$ (here we employ cgs units but give resistances in SI units). In quantum physics, the product of the fine structure constant $\alpha=$ $e^{2} / \hbar c=1 / 137.04$ and $c$ gives the conductance $e^{2} / \hbar$ (which is $2 \pi$ times the quantum unit for the Hall conductance).

The usual Hall effect, originally discovered in 1879 and by now a standard characterization technique for semiconductors, occurs when a magnetic field is applied, say, in the $z$ direction perpendicular to the current I (parallel to $x$ ), in a conductor. A Lorentz force operates in the $y$ direction and leads to a current in that direction for an electric field $E \| \hat{x}$ even in a uniform isotropic system. [In the usual experiments, with an open circuit $I_{y}=0$ an electric field $E_{y}$ related to the "Hall voltage" is generated.] Thus, the relation between the current density and electric field vectors is tensorial and the $x y$ component of the conductivity tensor is called the Hall conductivity. The $x y$ component of the inverse resistivity tensor is the Hall resistivity which is shown in the classical case to be given by $\rho_{x y}=-B / n e c, n$ being the density of carriers and $e$ their charge. Thus, in the simplest case the Hall effect provides a useful measure of the carrier density. In real experiments, one is measuring conductance, $G$, not conductivity, $\sigma$. The 2D case is special in that $\sigma_{x y}=G_{x y}$.

The regular conductance $G_{x x}$ of a MOSFET can be made very small in a large magnetic field at low temperatures $\left.{ }^{6}\right)$ by changing the gate voltage so that the Fermi level is between Landau levels. This means that the $j$ lowest Landau levels are full and all those from the $(j+1)^{\text {th }}$ on are empty. Thus, one has an effective gap of $\hbar \omega_{c}$ in the excitation spectrum and the system is insulating or dissipationless in the sense that $G_{x x}$ is exponentially activated $\left(\hbar \omega_{\mathrm{c}} \cong 6 \mathrm{meV}\right.$ and $T=1.5 \mathrm{~K}$ in the experiment of Ref. 1). Indeed, in the experiment conducted by von Klitzing at the Grenoble high field laboratory, $G_{x x} \leq 10^{-10}(\Omega)^{-1}$ was achieved over significant ranges of gate voltage $V_{g}$, accompanied by observable quantized plateaux in $G_{x y}$. This discovery was not sheer luck; it took an alert and astute observer, with a good deal of intuition and understanding of the physics involved, to grasp that this was a real and an extremely significant effect!

The effect was then interpreted in terms of the classical result for $\rho_{x y}$ (excluding spin and valley degeneracy ef fects). One recalls ${ }^{1}$ ) that the degeneracy per unit area of each Landau level is $p=H / \phi_{0}, \phi_{0}$ being the (single electron) flux quantum, $\phi_{0}=h c / e \cong 4 \times 10^{-15}$ $\mathrm{Tm}^{2}$. Thus, the surface electron density is $n=j p$, which yields Eq. 1. This explanation gives the essence of the effect. However, to understand the finite plateaux, one has to understand why the Fermi level remains pinned between Landau levels over finite ranges of electron density (or $V_{g}$ ). As recognized already in Ref. 1, this has to do with the existence of localized electronic states (due to unavoidable disorder). This introduces the fundamental problem of understanding localization and delocalization of states in 2D for large magnetic fields. In spite of many interesting subsequent developments, we still do not

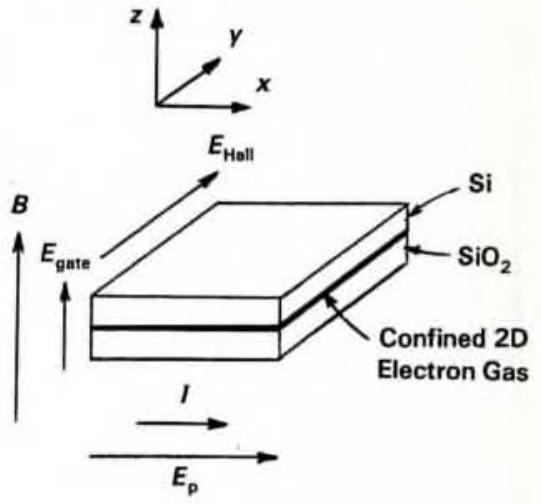

Fig. 2 - Schematic of the MOSFET showing the different field configurations.

have a quantitative understanding of the extent of the quantized Hall steps. Nor do we have a proper microscopic quanturm theory of the Hall effect for strong disorder.

\section{Developments and their Significance}

Since the velocity of light is a fixed quantity and is known with very good accuracy, the QHE means that one can now accurately measure the fine structure constant "with an ammeter and a voltmeter". Alternatively, knowing $e$ one can likewise measure Planck's constant!

The metrological consequences of this were fully realized in Ref. 1. By now, the method has already given a theory independent determination of $\alpha$ which is better than previous such determinations by aimost an order of magnitude. For producing resistance standards it is in principle absolute, time independent and relatively available.

Of course, such applications rest on the accuracy of Eq. 1. Does it have small corrections arising from solid state effects, higher order QED terms and relativistic effects? The last of these should not be very important because of the small $v / c\left(<10^{-9}\right)$ values usually used; solid state effects could be expected to be much larger than the measured accuracy of Eq. 1, so the experiments suggest that they are not relevant. Higherorder QED terms present an interesting open problem. Clearly, one needs a general theoretical argument, perhaps similar to that given by Bloch ${ }^{8}$ ) for the accuracy of determining $h / e$ from the Josephson effect, to explain the accuracy of Eq. 1.

Such an argument was indeed put forward by Laughlin ${ }^{9}$ ). In a simplified version the following Gedanken experiment is considered: The 2D system with the large magnetic field always perpendicular to the layer is wrapped on a cylinder and the small Hall voltage is generated by applying a Aharonov-Bohm flux, $\phi$, 
confined to the cylinder's opening. If $\phi$ varies linearly (adiabatically, for small enough $V): \phi=-c V t+$ const, an azimuthal EMF, $V$ is induced. We also consider the system to behave as a perfect insulator with $G_{x x}=0$, so it is in equilibrium at zero temperature. By very general arguments ${ }^{8}$ ), the states of the system are invariant to changing $\phi$ by a flux quantum $\phi_{0}$ (which is equivalent to changing the phase of the ground state wave function around the cylinder by $2 \pi)$. Thus each time $\phi$ changes by $\phi_{0}$ the only consequence is that an integral number of electrons, $j$, is transferred from the lower to the upper contact of the cylinder. This yields a current $l=j$ $e^{2} V / h$, consistent with Eq. 1! While not being able to calculate $j$ lexcept for free electrons) this argument establishes the generality and exactness of Eq. 1 within non-relativistic quantum mechanics. The current $l$ is a sort of supercurrent (since it flows perpendicular to $V$, there is no dissipation). The $\mathrm{QHE}$ is a macroscopic quantum phenomenon related to the fundamental role of the phase in quantum mechanics and its sensitivity to the vector potential (the AharonovBohm effect). In fact, the QHE has stimulated a recent revival of the interest in such effects in small normal conductor rings, where impressive results have recenty been reported.

A new surprise was in store for the theoreticians when experiments reported ${ }^{5}$ ) in 1982 in high quality quantum well samples first revealed a new Fractional Quantized Hall Effect (FOHE) in which the integer in Eq. 1 is replaced by a simple rational fraction such as $1 / m, m$ being an odd integer, as well as e.g. $2 / 3$, $2 / 5,2 / 7,3 / 7,4 / 5$, with more to come. This requires extra stabilization of the $2 \mathrm{D}$ electron gas at those fractional fillings of a Landau level, with gaps in the excitation spectrum. The relevant quasiparticles have fractional charges (a subject of current excitement in both solid state physics and quantum field theory) to agree with the above general argument. In fact, in a seminal theoretical paper, Laughlin ${ }^{10}$ ) showed how electron-electron Coulomb interactions can lead to such effects. The study of the 2D quantum plasma, has since become an important field of many body theory.

The universal significance of the QHE is further demonstrated by the usefulness of the concepts originating in it in high-energy physics. This is yet another demonstration (following the renormalization group method - 1982 Nobel Prize to K. Wilson) of how concepts developed in condensed matter con texts can become relevant, and even

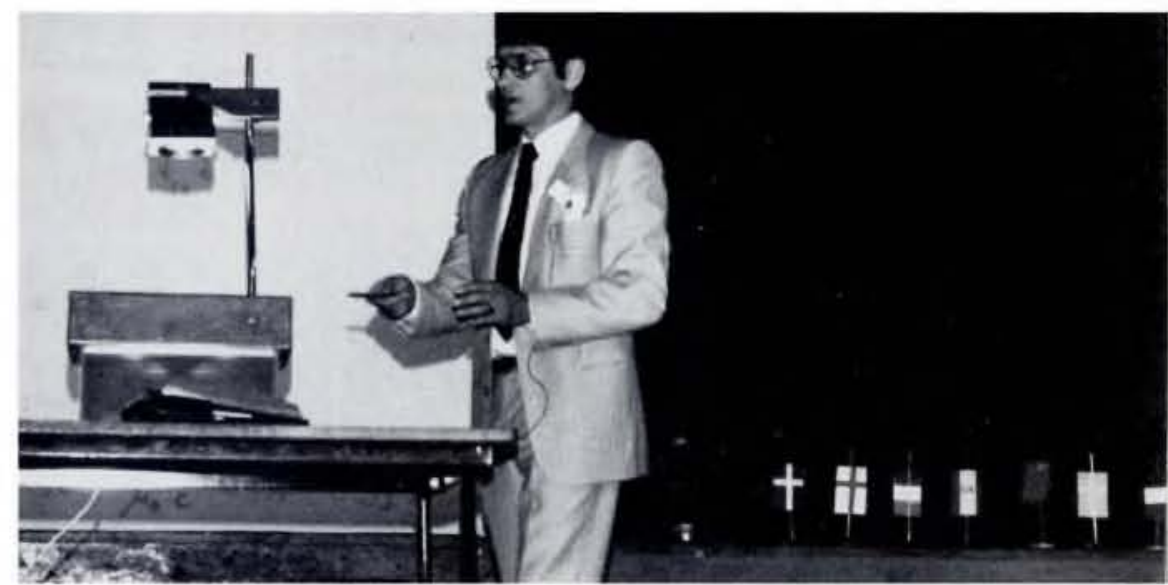

crucial, in other branches of physics. It is safe to predict that we shall be hearing even more on 2D electron systems and the $\mathrm{OHE}$ and related phenomena in the years to come.

\section{REFERENCES}

1. Von Klitzing K., Dorda G. and Pepper M., Phys. Rev. Lett. 45 (1980) 494

2. Kawaji S. and Wakabayashi J., in Physics in High Magnetic Fields, Eds. S. Chikazumi and N. Miura (Springer, Heidelberg) 1981.

3. Pepper M., Europhysics News, 16 (1985) $7 / 8$

4. Ando T., Matsumoto $Y$. and Uemura $Y ., J$. Phys. Soc. Japan, 39 (1975) 279.

5. Tsui D.C., Störmer H.L. and Gossard A.C., Phys. Rev. Lett. 48 (1982) 1559

6. Ando T., Fowler A.B. and Stern F., Revs. Mod. Phys. 54 (1982) 437

7. Fowler A.B., Sang S.S., Howard N.E. and Steines N.P.A. , Phys. Rev. Lett. $16(1966$ 901.

8. Bloch F., Phys. Rev. Lett. 21 (1968) 1241 Byers N. and Yang C.N., Phys. Rev. Lett. 7 (1961) 46 .

9. Laughlin R.B., Phys. Rev. B23 (1981) 5632; Halperin B.I., Phys. Rev. B25 (1981) 2185: Imry Y., J. Phys. C16 (1983) 3501 10. Laughlin R.B., Phys. Rev. Lett. 50 (1983) 1395

\section{CMD Applauds}

It is with great joy that the members of the EPS Condensed Matter Division learned the news that the 1985 Nobel Prize for Physics had been awarded to Dr. Klaus von Klitzing.

Von Klitzing is well known to EPS as he was awarded the EPS HewlettPackard Europhysics Prize* for 1981 and was presented with this award at the 2nd General Conference of the CMD held in Manchester in March 1982. His research and subsequent work have been regularly followed up at the CMD General Conferences and he was a plenary speaker at the 6 th General Conference held in Prague in August 1984 (when the above photo was taken).

The QHE (von Klitzing's and the fractional), despite much progress over the past few years presents a major challenge to theoretical physics.

* Europhysics News 13 (1982) 4.

\section{J.T. Devreese}

(Chairman of the Condensed Matter Division)

\section{THE PENNSYLVANIA STATE UNIVERSITY Experimental Low Temperature Physics}

The Department of Physics is seeking candidates for a tenure-track position of Assistant or Associate Professor in experimental low temperature physics starting in the $1985-86$ or 1986-87 academic years. Candidates should have an established record of research accomplishments and may expect to work in conjunction with the ongoing research effort on the main campus at University Park. Minimal requirements include a Ph.D. degree in this field and some postdoctoral experience. A desire and aptitude for teaching undergraduate and graduate students is essential.

Send applications, including a curriculum vitae and names of at least four references, to

Gerald A. Smith, Head, Department of Physics,

Box QQ, 104 Davey Laboratory. University Park, PA 16802, USA, by February 1, 1986 or until a suitable pool of applicants is identified.

An affirmative action/equal opportunity employer. 